\title{
EVALUATING THE MECHANICAL RESPONSE OF MATERIALS AGAINST SMALL IMPACT FORCE
}

\author{
Irfa Aji Prayogi ${ }^{\mathrm{a}}$, Kazuhide Watanabe ${ }^{\mathrm{a}}$, Kyohei Irisa $^{\mathrm{a}}$, Akihiro Takita $^{\mathrm{a}^{*}}$, Mitra Djamal ${ }^{\mathrm{b}}$, Yusaku Fujii $^{\mathrm{a}}$ \\ ${ }^{\text {aS }}$ School of Science and Technology, Gunma University \\ 1-5-1 Tenjincho, Kiryu 376-8515, Japan \\ bepartment of Physic, Faculty of Mathematics and Natural Sciences, Institut Teknologi Bandung, \\ Jalan Ganesa 10, Bandung 40132, Indonesia \\ *Corresponding Author: t13802281@gunma-u.ac.jp
}

\begin{abstract}
This paper deals with mechanical response of material against small impact force using levitation mass method (LMM). Material properties observed by evaluate deformation of material and force acting on the material. In LMM, the force acting on the material is generated by collision of a levitated mass which has very small friction. The impact Force and deformation of material which cause of the collision is accurately measured by optical interferometer. A dynamic impact force, less than $10 \mathrm{~N}$ are applied to the three types of material. The mechanical response of material is indicated by time of collision, $\mathrm{W}_{\mathrm{hv}}$ and ratio of loss energy. It shows stiffness properties and elasticity level of material, respectively.
\end{abstract}

Keywords: Levitation mass method, Dynamic impact force, Material testing, Mechanical responds.

\section{Introduction}

The general method used for material testing that is evaluating a force that applied to a material using a force transducers and the deformation of material (1-3). This method depends with measurement of force transducers and position transducers. At present, force transducers are typically calibrated using static weights and under static conditions, are widely available. Methods for the dynamic calibration of force transducers are important for fulfilling these requirements. Force measures as inertial force of mass which role as reference in force calibration or material testing. There is some attempt to developing the dynamic method for generating and measuring a force $(2,3)$. However, there are no accepted standard methods available for dynamic method for force calibration and material testing at present. Kumme proposed a method which uses the inertial force of the attached mass generated by a shaker (2). Fujii has developed a method that well known as Levitation Mass Method $(3,4)$. In this method, the inertial force is generated by a levitated mass (moving part) which collides to material or force transducers under tested. The Force generated manually with pushing the moving part. The moving part adjusts to have a linear motion and very small friction using a pneumatic linear bearing (5). The inertial force of the moving part is measured using an optical interferometer.

In recently research, some experiment using LMM for material testing was conducted. An evaluating material viscoelasticity using an oscillation inertial force was clearly described (6). Strength test for general industrial product was conducted using impact force as reference force (7). A big capacity of linear bearing that have $30 \mathrm{~kg}$ in maximum weight was used to generate a big amount of inertial force. Moreover, a material testing in micro-level force was also observed. The measured force acting on the mass is approximately $0.1 \mathrm{mN}$. The micro-Newton level force generated using a small capacity of linear bearing (8). Using this method, mechanical response of a piece of paper has been observed with micro-Newton level of impact forces, whose maximum value was approximately $2 \mathrm{mN}$. However, the evaluation a dynamic mechanical response for the stiff material in small impact force has not been conducted.

This paper describes an evaluating of mechanical response of solid material against impact force using a levitation mass method. 


\section{Experimental Setup}

Figure 1 shows a schematic diagram for evaluating dynamic properties of the material. A material under test was firmly attached at the base. Then, an impact force applied to the material by colliding the moving part of aerostatic linear air bearing, which had a mass $17.85 \mathrm{~g}$. The moving part collided to the material being tested with initial velocities, $v_{i}(\mathrm{~m} / \mathrm{s})$ that applied by pushing manually. After the collision, the moving part reflected with decelerates velocities; it measures as velocities after collision, $v_{f}(\mathrm{~m} / \mathrm{s})$.

While the collision, the velocity of moving part accurately measured by optical interferometer system. The optical interferometer system has one corner cube prism which firmly attached to moving parts; it defined as the signal arm. The other corner cube prism was at rest; it defined as the reference arm.

The light source of the optical interferometer is a Zeeman-type He-Ne Laser. It emits a light at two wavelength in which has orthogonal polarization. The light from laser is incident on a non-polarization beam splitter (NPBS). The reflected beams would be through a Gland-Thomson Prism (GTP) then the interfered beam is directly measured by photo detector as rest signal. The measured frequency from rest signal is defined as $f_{\text {rest }}$. The transmitted beams were incident on the polarization beam splitter (PBS) and would be separated as polarization state. One of the beams transmitted to signal arm and the other beam reflected to reference arm. Before both of a beam reflected by the corner cube, it had been through a quarter wave plate in order to rotate the polarization state. Then both of beams would propagate in reference arm. After propagation in the Michelson interferometer arms, the two beams made to interfere by transmitting them through a GTP. The interfering beams were go to photo detector and resulted a beat signal. The measured frequency beat signal, $f_{\text {beat }}$, which corresponds to the frequency difference between the signal and reference beams, measured with the digitizer. When the moving part was at rest, the $f_{\text {beat }}=f_{\text {rest }}$ was approximately $1.8 \mathrm{MHz}$.

While the moving part moves, the beat frequency would be varies but still around rest frequency value. It depends the velocity of moving part. The velocity is calculated as the Doppler shift frequency of the signal beam of the laser interferometer $f_{\text {Doppler, }}$, which expressed as

$$
\begin{aligned}
& v=\frac{\lambda_{\text {air }} f_{\text {Doppler }}}{2}, \\
& f_{\text {Doppler }}=-\left(f_{\text {beat }}-f_{\text {rest }}\right),
\end{aligned}
$$

where the $\lambda$ air is the wavelength of the laser beam which is approximately $632.8 \mathrm{~nm}$. The positive direction for the position, velocity, acceleration and force acting on the moving part is towards the right in figure 1 .

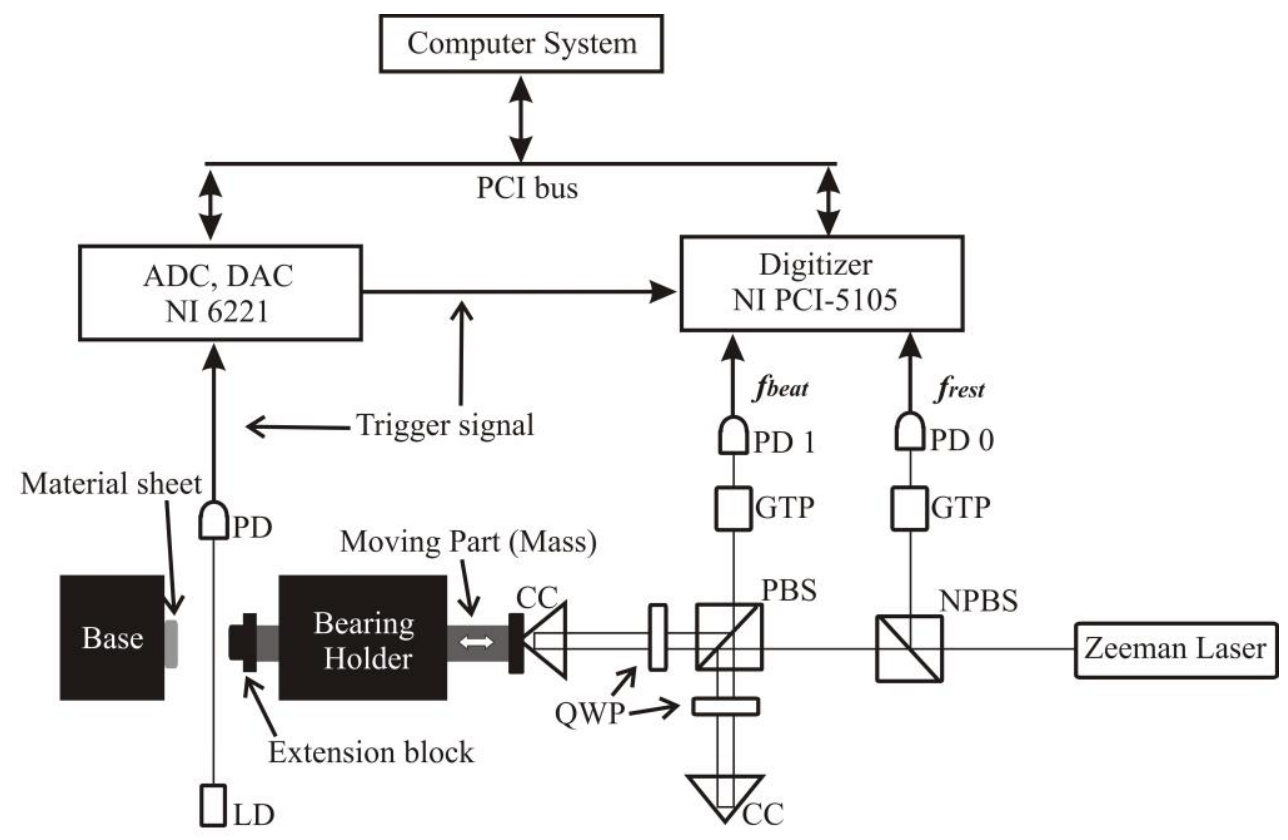

Fig. 1. Experimental setup. $\mathrm{PD}=$ Photo Diode, $\mathrm{LD}=$ Laser Diode, $\mathrm{ADC}=$ Analog-to-Digital Converter, $\mathrm{DAC}=\mathrm{Digital}-\mathrm{to}-$ Analog Converter, $\mathrm{CC}=$ Corner Cube, $\mathrm{QWP}=$ Quarter Wave Plate, GTP $=$ Gland-Thompson Prism, PBS = Polarizing Beam splitter and NPBS $=$ Non-Polarizing Beam splitter. 
There are 3 different kind materials has been tested, i.e. Urethane, Silicon rubber and Low elasticity rubber (Manufactured by Misumi). Photograph of the material under tested is shown in Fig. 2. All material was firmly attached in base using double tape. All sheet was cut with size of $18 \times 15 \times 1 \mathrm{~mm}$. A digitizer (model: PCI-5105; manufactured by National Instruments Corp., USA) recorded both signals from PD0 and PD1 with a sampling number of $5 \mathrm{M}$ samples for each channel and a sampling rate of $30 \mathrm{MS} \mathrm{s}-1$. The measurement duration of the digitizer is $0.25 \mathrm{~s}$.

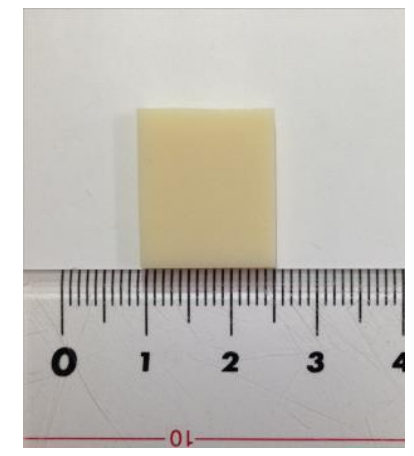

(a) Silicon rubber

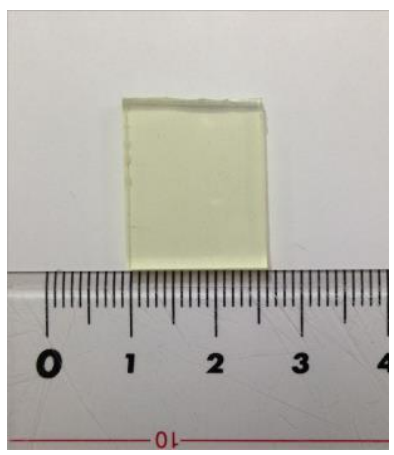

(b) Urethane

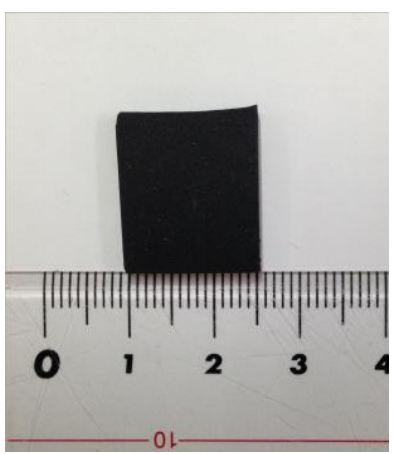

(c) Low elasticity rubber

Fig. 2. Photograph of rubber sheet under tested

The pneumatic linear bearing, "GLS08A50/25-2571" (NSK Co., Ltd., Japan), was attached to an adjustable tilting stage. The maximum weight that supported by the moving part is approximately $1 \mathrm{~kg}$.
The stroke of the movement is approximately $25 \mathrm{~mm}$ and the thickness of the air film is approximately $10 \mu \mathrm{m}$. The tilting angle of the tilting stage was adjusted horizontally with the uncertainty of approximately $0.1 \mathrm{mrad}$. Measurements in digitizer triggered by a sharp step signal generated using a digital-to-analogue converter. This trigger signal is initiated by a light switch, when the moving part covered the light of laser diode to photodiode.

In the testing, 35 collisions were conducted by manually changing the initial velocity of the moving parts for each material.

\section{Result and Discussion}

Figure 3 shows the data processing procedure. During the test, only frequency was highly accurately measured using an optical interferometer system. The Doppler shift frequency is measured as the difference between the beat and rest frequency. The velocity, position, acceleration and the force of the moving part are generated from the frequency afterward. The origin of the zero point axis is set where the reaction force from the material under test is detected. The first time collision is a first point of ascending series from six sequentially points in force data. Then the first point of the series is used as an origin of the zero point axis.

In Fig. 4, 5, and 7 show that dynamic mechanical properties of all material collisions, it has a maximum force collision less than 10 N. Fig. 4 and 6 shows the same experiment for each material. In Figure 4 shows the measured force using optical interferometer for each material. It was choose that has a similar maximum force at around $3.5 \mathrm{~N}$ each material. It shows the collision time of materials. The silicone rubber had the longest time than the other. The collision time is represented by full width half value maximum (FWHM), $W_{h v}$ of the force. $W_{h v}$ of the silicone rubber, urethane, and low elasticity rubber is $1.37 \mathrm{~ms}, 0.62$ $\mathrm{ms}$, and $0.34 \mathrm{~ms}$, respectively.

The relationship of $W_{h v}$ and force mainly comes from the mechanical properties such as viscosity and elasticity of the materials. The range of $W_{h v}$ for silicon rubber, urethane and low elasticity rubber shows in Fig. 5. It is 1.34 to 1.71 $\mathrm{ms}, 0.44$ to $0.67 \mathrm{~ms}$, and 0.25 to $0.35 \mathrm{~ms}$, respectively. The $\mathrm{W}_{\mathrm{hv}}$ decreases according to increasing of maximum force for all materials. 

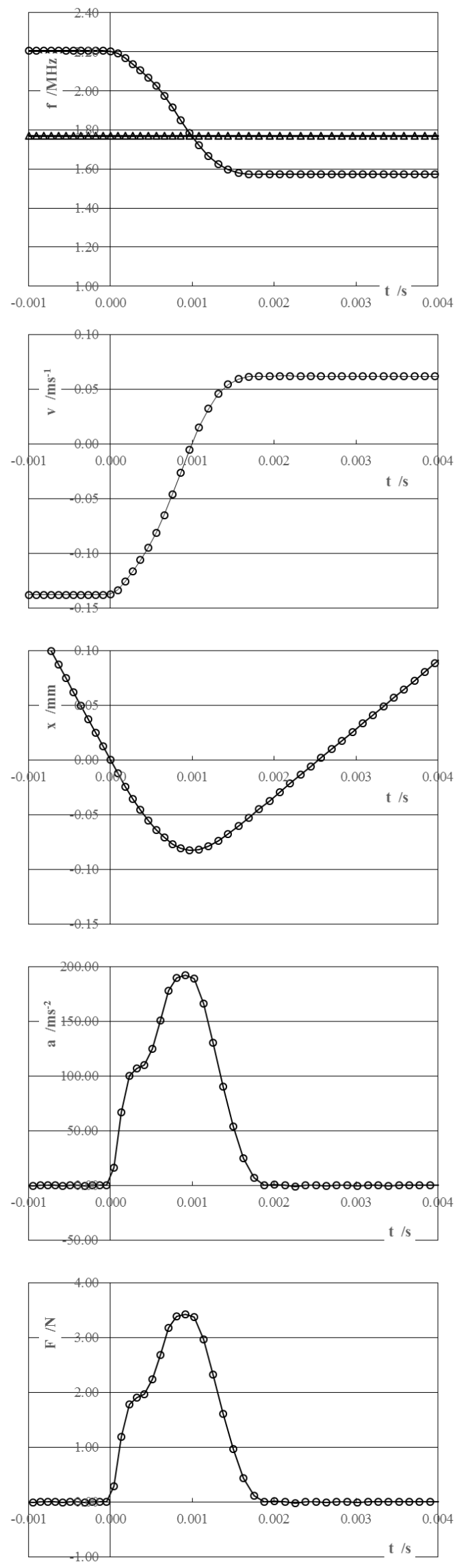

Fig.3. Data processing procedure
Figure 6 shows the change in normalized force against position. Normalize force is calculated as measured force/Force mass, max. Fig. 6 shows the silicon rubber pressed more depth in the same force, its indicate softness of the material. The relationship of the change force against the change of position for all collision shows in Fig. 7 the average of the spring constants, $k$ of silicon rubber is 12.17 $\mathrm{N} / \mathrm{m}$, urethane $97.97 \mathrm{~N} / \mathrm{m}$, and for the low elasticity rubber is $110.06 \mathrm{~N} / \mathrm{m}$, respectively.

The stiffness of material showed with the longer Halfwidth value, the depth while pressed and the lowest the spring constant. It means the silicon rubber is softest material in this test. The force acting on the material from the mass is expressed as $\boldsymbol{- F}$ according to the law of action and reaction. The hysteresis of Force which is caused by the viscosity of the materials, is clearly observed. The area under the curve in Fig. 6 is equal with the absolute value of the work. This value is similar with the reduction or loss of kinetic energy.

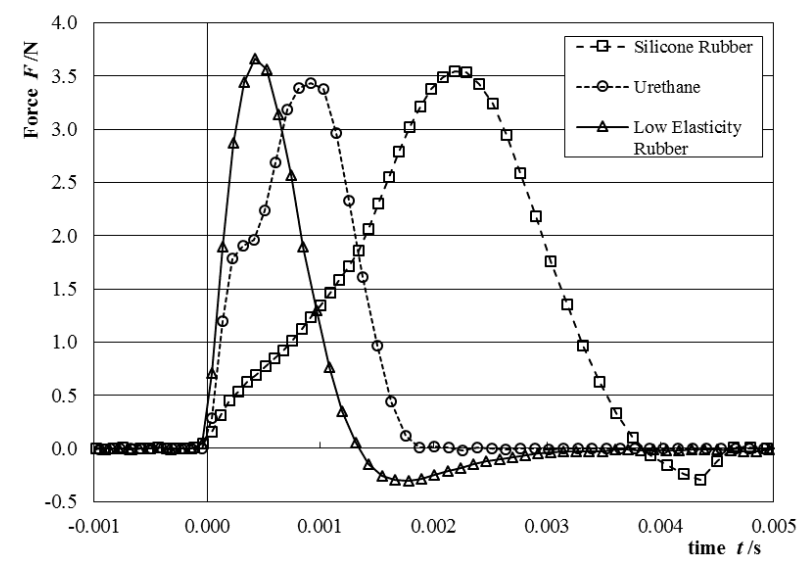

Fig. 4. Measured impact force against time

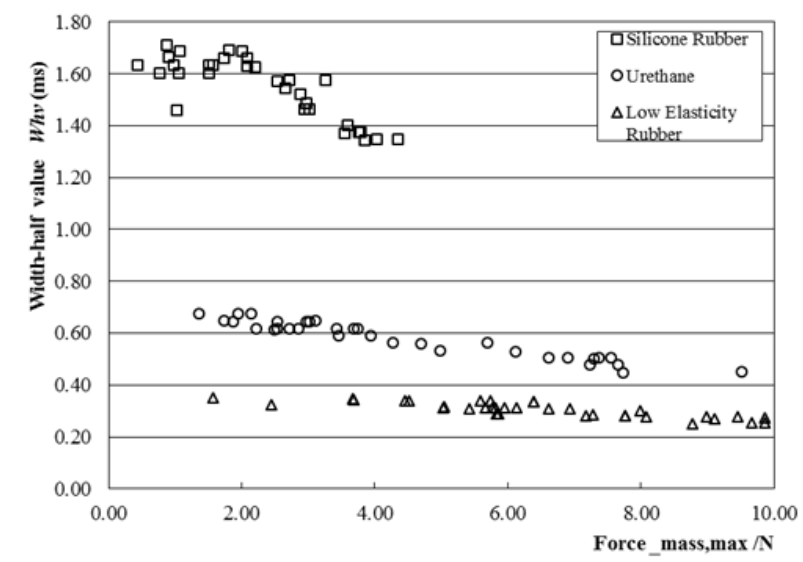

Fig. 5. Collision time (Full Width half Maximum) for all materials 
In the calculation of kinetic energy, $E k=1 / 2 m v^{2}$, only the velocity is influential, which is, $v_{i}$ and $v_{f}$. The reduction of the energy is calculated as the difference of kinetic energy before collision, $\mathbf{E}_{\mathbf{i}}$, against after collision, $\mathbf{E}_{\mathbf{f}}$. Loss of kinetic energy for silicone rubber, urethane and low elasticity rubber are approximately $4.31 \times 10^{-4}, 1.36 \times 10^{-4}, 1.48 \times 10^{-4} \mathrm{~J}$, respectively. The ratio of the dissipation energy, loss Energy/ $E_{i}$, each material shows in figure 8 . The ratio of loss energy in low elasticity rubber is the highest than the other at approximately $97.89 \%$ of initial kinetic energy. It means the low elasticity rubber absorbs bigger energy than the other materials. In the experiment, the low elasticity rubber could not restore the moving part to the starting point. It means the low elasticity rubber has a low-level elasticity. The level elasticity and stiffness showed by the ratio of absorbing

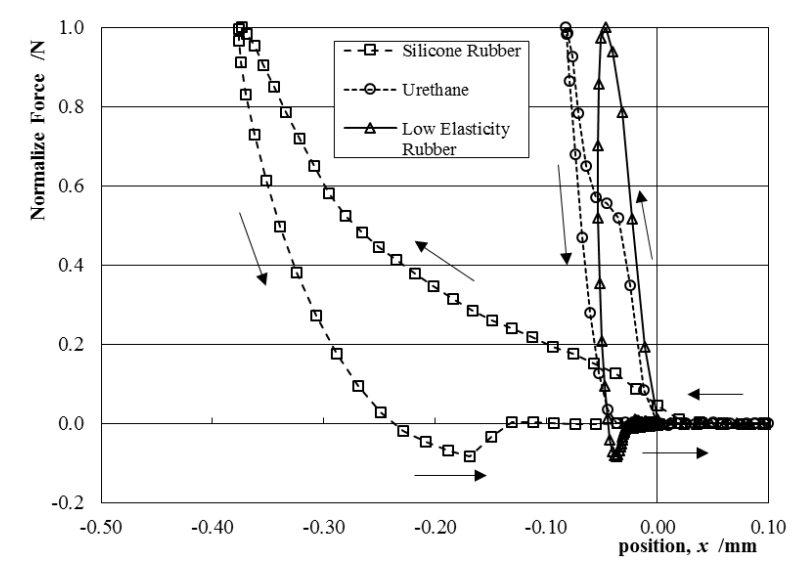

Fig. 6. Change of Normalize force against position

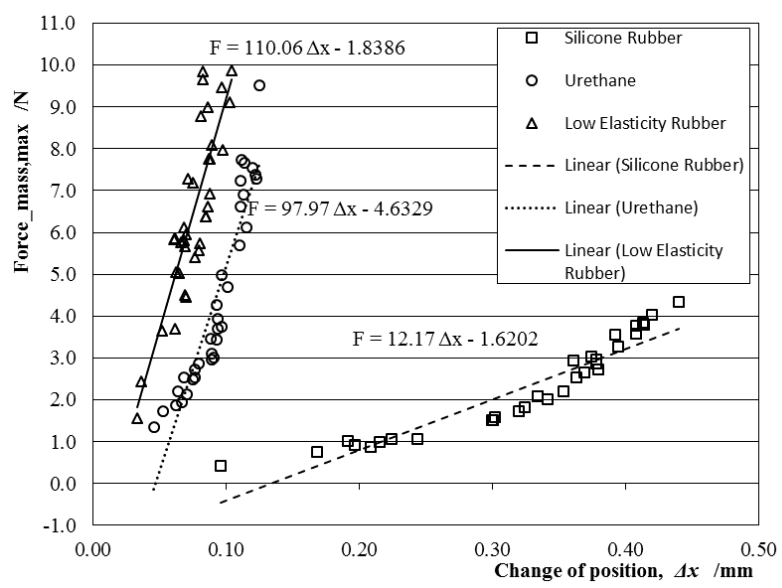

Fig. 7. Change of Force against change position all collision

energy by material, for the urethane and silicone rubber are approximately $73.40 \%$ and $74.31 \%$, respectively.

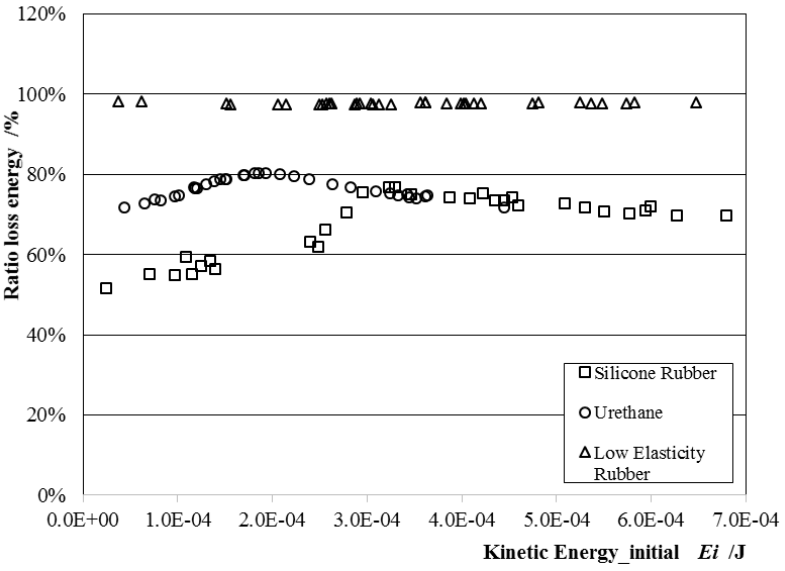

Fig. 8. Ratio of loss energy against initial kinetic energy

\section{Conclusions}

The evaluation of dynamic mechanical properties of material against small impact load has been carried out. The range of force is $1 \mathrm{~N}$ to $10 \mathrm{~N}$. In this test, stiffness value each material can be observed with notice of width-half value, the ratio of loss energy, and the spring constant.

\section{Acknowledgment}

This work was supported in part by a research-aid fund of the NSK Foundation for the Advancement of Mechatronics (NSK-FAM) and the Grant-in-Aid for Scientific Research (B) 24360156 (KAKENHI 24360156).

\section{References}

(1) Jan Peter Hessling : " Dynamic calibration of uni-axial material testing machines", Mechanical Systems and Signal Processing, Vol.22, No.2, pp. 451-466, 2008

(2) Rolf Kumme, and M.J. Dixon : " The result of comparisons between different dynamic force measurement system", Measurement, Vol.10, No.3, pp. 140-144, 1992

(3) Yusaku Fujii, and Hiroyuki Fujimoto : "Proposal for an impulse response evaluation method for force transducers", Vol. 10, No. 4, pp. N31-N33, 1999

(4) Yusaku Fujii : "Toward dynamic force calibration", Measurement, Vol. 42 No. 7, pp. 1039-1044, 2009

(5) Yusaku Fujii : "Measurement of force acting on a moving part of a pneumatic linear bearing", Review of Science Instruments Vol. 6, No. 74, pp. 3137-3141, 2003 
(6) Yusaku Fujii, and Takao Yamaguchi : "A method for evaluating material viscoelasticity", Review of Science Instruments, Vol. 75, No. 1, pp. 119-123, 2004

(7) Tomokazu Suzuki, Yusaku Fujii, and J.D.R. Valera : "Optical method for strength test for general industrial product", Mechanical Systems and Signal Processing, Vol. 20, No. 3, pp. 735-744, 2006

(8) Yusaku Fujii : "Method for generating and measuring the micro-Newton level forces", Mechanical Systems and Signal Processing, Vol. 20, No. 6, pp. 1362-1371, 2006

(9) Yusaku Fujii : "Microforce materials tester", Review of Science Instruments, Vol. 76, No. 065111, 2005 